\title{
Development of a Software Package for Cattle Identification in Nigeria
}

\author{
*1BELLO, RW; ${ }^{2}$ ABUBAKAR, $\mathrm{S}$ \\ *l Department of Mathematical Sciences, Faculty of Basic and Applied Sciences, University of Africa, Toru-Orua, Bayelsa State, Nigeria \\ ${ }^{2}$ Department of Mathematics, School of Science Education, Federal College of Education (Technical) Bichi, Kano State, Nigeria \\ *Corresponding Author Email: sirbrw@yahoo.com
}

\begin{abstract}
Open grazing or free-range grazing is one of the methods employed by the Nigeria nomadic cattle herders to provide pasture for their cattle. This method of providing pasture for cattle comes with so many challenges among which are cow swapping, ownership disputes, rustling and cow intrusion to farmland. Some existing methods of guiding against these challenges are expensive, injurious, and unreliable to apply. The objective of this paper is to develop an enhanced and affordable software package for cow recognition and identification using a graphical user interface and information encoding method. Data analysis module with software application for the analysis of the generated code is proposed; the software application installed on a computer or smart-phone may be standalone or otherwise. Data about individual cow is digitally collected, coded and stored using necessary resources, tools, and methods. Moreover, by tagging individual cow with the generated code, and matching the code with the ones in the database using code reader, individual cow can be recognized and identified.
\end{abstract}

\section{DOI: https://dx.doi.org/10.4314/jasem.v23i10.9}

Copyright: Copyright (C) 2019 Bello and Abubakar. This is an open access article distributed under the Creative Commons Attribution License (CCL), which permits unrestricted use, distribution, and reproduction in any medium provided the original work is properly cited.

Dates: Received: 28 September 2019; Revised: 22 October 2019; Accepted 26 October 2019

Keywords: Open grazing; Free-range grazing; Nomadic herder; Cow identification; Pasture.

So many reasons are responsible for the open and freerange grazing method employed by the nomadic cow herders. Sometimes this is due to the shortage of pasturage and water which are essentially required for cow sustenance. The unavailability of pasturage and water sometimes leads to a nomadic approach of moving from one location to another in search of pasture. This nomadic method of grazing sometimes leads to cow getting strayed, rustled, swapped, and intruded to farmland causing disputes, destruction, and even death. To address these challenges and many others, different animal recognition and identification methods have been proposed (Kumar et al., 2019; Kumar et al., 2018; Bello, 2018; Cheema and Anand, 2017). However, most of these recognition and identification methods are expensive, injurious, unreliable and sometimes, cause death. The need to profound cheap, injury-free and reliable method of cow recognition and identification becomes a necessity. The objective of this paper is to develop an enhanced and affordable software package for cow recognition and identification using a graphical user interface and information encoding method. Data analysis module with software application for the analysis of the generated code is proposed; the software application installed on a computer or smartphone may be standalone or otherwise. Data about individual cow is digitally collected, coded and stored using necessary resources, tools, and methods. Moreover, by tagging individual cow with the generated code, and matching the code with the ones in the database using code reader, individual cow can be recognized and identified.

Many researchers have contributed to the animal identification project. Most of the recent works on animal identification use deep learning or a novel approach. While there are lots of efforts to automatically identify animals in camera-trap images; a lot of people still depend on hand-designed features (Swinnen et al., 2014, Figueroa et al., 2014).

Norouzzadeh et al. (2018) applied deep learning for the extraction of essential features in their animal identification project. They made use of a large dataset of wild animals for the application of their method. Apart from camera-trap, hand-designed, and sparse coding spatial pyramid matching (Yu et. al., 2013) methods of extracting features for identification, many other recent recognition and identification projects that employed deep learning include Zin et al. (2018) and Seijas et al. (2019).

For better identification, many researchers have employed animal biometrics using muzzle point or cow nose point for cow identification (Kumar et al., 2018). The essential features of the muzzle point were captured using camera, paper-based or ink nose print methods and processed using the modern technique. The similarity scores between the muzzle print images 
and the ones in the database were calculated using the scale-invariant feature template (SIFT). However, there is a need to enhance cow recognition and identification methods currently employed.

\section{METHODOLOGY AND RESULT}

Presented in this section is the proposed cow identification system (Fig. 1); this comprises of four components with one of the components representing the system (core component) and the remaining three components representing the subsystems, all embedded in the identification system. The core component is responsible for providing the functionalities needed by the entire system among which is the graphical user interface (Fig. 2) that is split into some parts among which is the registration part. Stored basic information about the cows, the herders and the premises where the cows are kept is digitally captured, processed and converted to software code, printed and permanently tagged on individual cow for identification (Fig. 3). For cow recognition and identification, the image of the printed code tagged on the individual cow is captured and scanned with code reader for recognition and identification purposes.

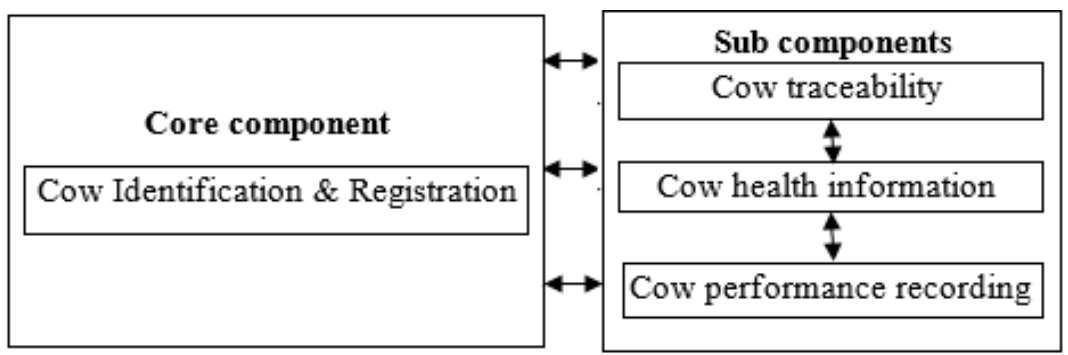

Fig. 1: Components of Cow Identification System

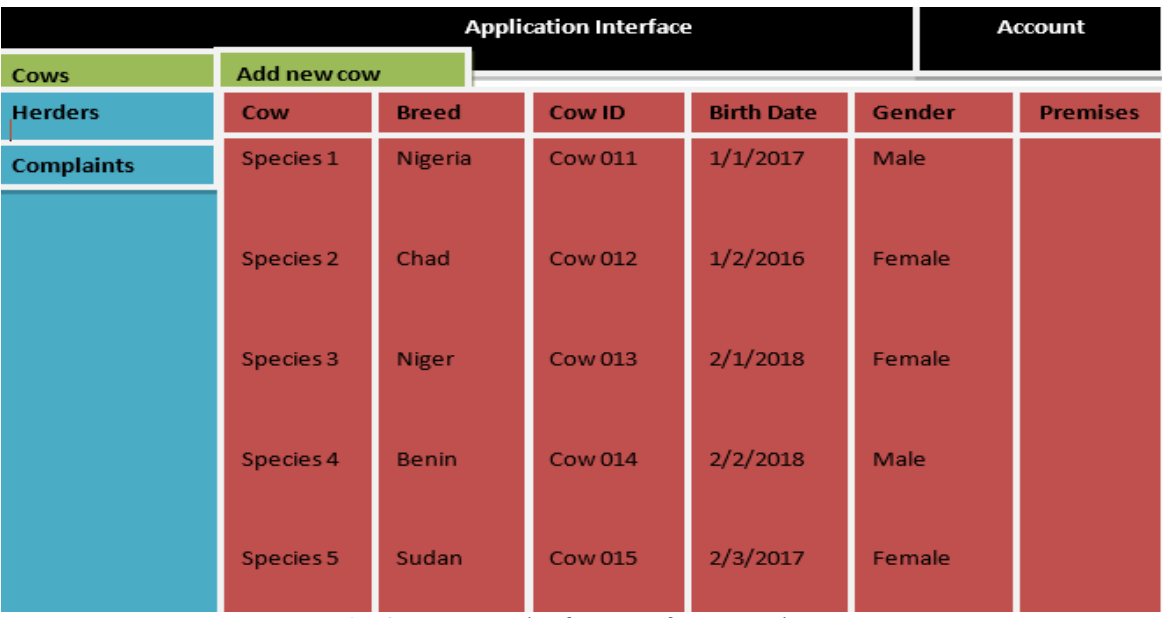

Fig. 2: Framework of GUI Software Package

Software and Graphical User Interface (GUI) Design: The component of the system which is the core part provides the functionality that is shared with the remaining subcomponents, thereby reducing the overhead cost that would have been incurred implementing the functionalities expected from the remaining subcomponents. The core component is responsible for providing the functionalities needed by the entire system among which is the graphical user interface (Fig. 2) that is split into some parts among which is registration part. Stored basic information about the cows, the herders and the premises where the cows are kept is digitally captured, processed and converted to software code, printed and permanently tagged on individual cow for identification (Fig. 3).

Identification and registration of individual cow: Irrespective of the features used for cow identification; a device and identification code appropriate for the individual cow and the reason for applying the methods are important. Taking, for example, if the reason for identification and registration is to affirm ownership of cows in a community, a brand may be all that is required. For animal like cow, tagging them with a group code that represents group movements and the premises where they are being reared may be sufficient for their identification. Also, an unparalleled 
lifetime identification code may be all that is needed for individual cow's activity and performance to be monitored. The primary purpose of the identification code is to connect the cow to the premises where it is kept. Whenever individual cow is to be identified, specific information about the cow must be captured and taken care of at the premises, including those in the database. Among the minimum data required for capturing during the registration process for unique identification of individual cow are cow id, breed, sex, year or date of birth and premises id (location of birth).

Developing a unique identification numbering system for individual cow: Numerically, identification code is programmed to be short in length and it is expected of it to uniquely be available for a turnover period of at least 5 decades for the cow. Wherever pedigree information is stored in cow breeding, a more prospicient turnover period is needful. Cow identification code that is simple and short is difficult to forget and will be very easy to handle. Nonetheless, cow identification code often contains additional information such as cow number and area. This inclusion of additional information to cow identification code can reveal more useful information about the cow. Nonetheless, as important as this additional information is, it also extends the length of the cow identification code and substantially cuts down the number of possible cow identification code.

The management of identification code is rendered more complicated in structure and costly with the storage of a larger number of identification devices required. International Standard Organisation (ISO) 11784 is the established international standard for the structure of identification codes (FAO, 2016). The reason for developing the structure is for its worldwide use in electronic identification, though, it can also be applied in non-electronic identification codes. It has basic structure of an individual animal identification code which consists of the country code followed by a 12 digits number. The country code is usually made up of two digits alpha code used for the traditional identification (visual tags) of individual and numerically formed three digits ISO 3166 code used for electronic identification. With this, harmonizing format of identification devices worldwide is made possible. The last digit of the 12 digits number in most cases is used as a check digit. This code works by digitally scanning the tagged code using scanner or reader to reveal the identity of the cow.

Algorithm method to calculate a check digit: In 12 digits number, a check digit is a function of the first 11 digits. The primary use of check digit is to prevent false animal identification code entry. The algorithm is used to compute whether the check digit is correct when the animal identification code is read. Check digit will not match the computation if any digit is typed incorrectly and a message will be prompted by the software that the animal identification code is wrong. To ensure that the correct data is applied to the correct animal, the correct identification code may then be entered.

Let us take for example that a tag number is 123456789105, where 1 is the first digit, 2 is the second digit and 0 is the eleventh digit. The check digit is the last digit; this is derived from the first 11 digits as follows: The first digit is multiplied by 11 , the second by 10 , the third by 9 and, lastly, the eleventh by 1 . Then, 9 is used to divide the sum total of these numbers. The check digit, therefore, is the remainder of this sum. For the tag number 123456789105, the check digit is 5 , this is derived as follows:

$(1 \times 11+2 \times 10+3 \times 9+4 \times 8+5 \times 7+6 \times 6+7 \times$ $5+8 \times 4+9 \times 3+1 \times 2+0 \times 1) / 9)=257 / 9=28$ remainder 5 .

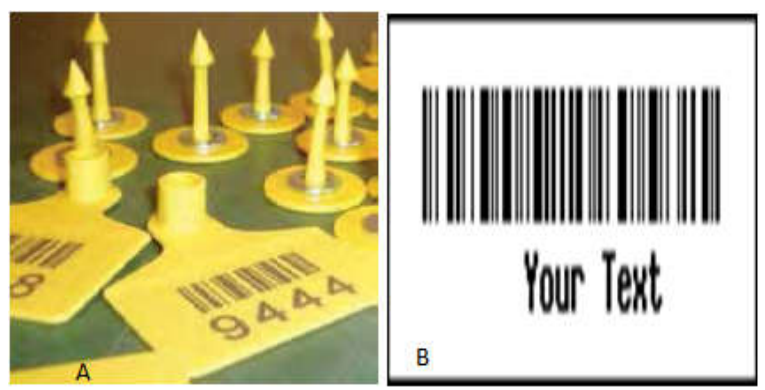

Fig. 3: (a) Sample of assigned code (b) Sample of unassigned code

Conclusion: This work has developed a framework for cow identification software in Nigeria using the algorithm method to calculate a check digit used in code matching. The software will reduce the incident of cattle rustling and cow intrusion to farmlands and aid animal health information and performance recording.

\section{REFERENCES}

Bello, RW (2018). An overview of animal behavioral adaptive frightening system. International $J$. Math. Physic. Sci. Res. 6 (1): 126-133.

Cheema, GS; Anand, S (2017). Automatic detection and recognition of individuals in patterned species. In: Joint European Conference on Machine Learning and Knowledge Discovery in Databases. 27-38. 
FAO (2016). Development of integrated multipurpose animal recording systems. FAO Animal Production and Health Guidelines. No. 19. Rome.

Figueroa, K; Camarena-Ibarrola, A; García, J; Villela, HT (2014). Fast automatic detection of wildlife in images from trap cameras. In: Iberoamerican Congress on Pattern Recognition, 940-947. Springer, Cham.

Kumar, S; Pandey, A; Satwik, KSR; Kumar, S; Singh, SK; Singh, AK; Mohan, A (2018). Deep learning framework for recognition of cattle using muzzle point image pattern. Measurement, 116: 1-17.

Kumar, S; Singh, SK (2019). Cattle Recognition: A New Frontier in Visual Animal Biometrics Research. In: Proceed. Nat. Acad. Sci. Section A: Physic. Sci. 1-20.

Norouzzadeh, MS; Nguyen, A; Kosmala, M; Swanson, A; Palmer, MS; Packer, C; Clune, J (2018). Automatically identifying, counting, and describing wild animals in camera-trap images with deep learning. Proceed. Nat. Acad. Sci. 115 (25): E5716-E5725.
Seijas, C; Montilla, G; Frassato, L (2019). Identification of Rodent Species Using Deep Learning. Computación y Sistemas. 23 (1): 257.

Swinnen, KR; Reijniers, J; Breno, M; Leirs, H (2014). A novel method to reduce time investment when processing videos from camera trap studies. PloS one, 9 (6): e98881.

Yu, X; Wang, J; Kays, R; Jansen, PA; Wang, T; Huang, T (2013). Automated identification of animal species in camera trap images. EURASIP J. Image and Video Processing. 1: 52.

Zin, TT; Phyo, CN; Tin, P; Hama, H; Kobayashi, I (2018). Image technology based cow identification system using deep learning. In: Proceedings of the International MultiConference of Engineers and Computer Scientists. 1. 